Tropical Journal of Pharmaceutical Research November 2017; 16 (11): 2595-2600

ISSN: $1596-5996$ (print); 1596-9827 (electronic)

(c) Pharmacotherapy Group, Faculty of Pharmacy, University of Benin, Benin City, 300001 Nigeria.

All rights reserved.

Available online at http://www.tjpr.org

Original Research Article

http://dx.doi.org/10.4314/tjpr.v16i11.4

\title{
Up-regulation of corticotropin releasing hormone is associated with the downregulation of corticotropin releasing hormone binding protein and up-regulation of IL- 33 and IL-8 expression in psoriasis
}

\author{
Fei Su, Yun Xia, Meng Huang, Liang Zhang and Liuqing Chen* \\ Department of Dermatology, Wuhan No. 1 Hospital, Wuhan, Hubei Province, PR China
}

*For correspondence: Email: chlq35@126.com; Tel/Fax: +862785332621

Sent for review: 28 July 2017

Revised accepted: 27 October 2017

\begin{abstract}
Purpose: To determine the expression of corticotropin releasing hormone (CRH) in psoriasis and normal skin biopsy samples, and to correlate the expression of CRH with the expression of CRHBP and inflammatory cytokines IL-8 and IL-33.

Methods: Psoriasis and normal skin biopsy samples were obtained from three psoriatic and three normal healthy patients. The mRNA expression was examined by quantitative real-time polymerase chain reaction (RT-PCR). Protein expression analysis was carried out by Western blotting and then further validated by immunohistochemistry.

Results: The results of the present study revealed that the expression of CRH was highly significant ( $p$ $<0.0001$ ) in psoriatic skin, compared to normal skin. Increase of CRH in psoriatic samples ranged from 2.7 to 3.5-fold. Expression of $\mathrm{CRH}$ was associated with the concomitant downregulation of CRHBP in all the psoriatic skin biopsy samples, with expression of CRHBP 3.0 to 6.2-fold lower in psoriatic skin than in normal skin. Analysis of mRNA expression of IL-8 and IL-33, revealed that expression of both IL-8 and IL-33 was significantly $(p<0.0001)$ upregulated in psoriatic skin samples while the expression of IL8 and IL-33 was approximately 4.1- and 3.2-fold higher in psoriatic skin than in normal skin. The expression of CRHBP, IL-8 and IL-33 was further confirmed by western blotting and immunohistochemistry results.

Conclusion: The results confirm that the expression of $\mathrm{CRH}$ is associated with the suppression of CRHBP and upregulation of IL-8 and IL-33.
\end{abstract}

Keywords: Psoriasis, Corticotropin releasing hormone, Inflammatory cytokines, Interleukin, Skin biopsy

Tropical Journal of Pharmaceutical Research is indexed by Science Citation Index (SciSearch), Scopus, International Pharmaceutical Abstract, Chemical Abstracts, Embase, Index Copernicus, EBSCO, African Index Medicus, JournalSeek, Journal Citation Reports/Science Edition, Directory of Open Access Journals (DOAJ), African Journal Online, Bioline International, Open-J-Gate and Pharmacy Abstracts

\section{INTRODUCTION}

Psoriasis is one of the chronic inflammatory skin disorders that affect around $3 \%$ of the human population around the globe. However, studies have reported that the incidence of psoriasis may vary with race and geographical locality and the environmental conditions [1,2]. Psoriasis has broad clinical spectra ranging from the involvement of skin to erythroderma [3]. Psoriasis has been categorized into two different types. Type I psoriasis is genetic, usually arises at or around the age of 16 years in females and 22 years in males. Type II psoriasis is sporadic and generally affects the people around 60 years of age [4]. The development of psoriatic plaques triggers pain and generates a lot of discomfort [5]. 
Psoriasis is accompanied by prolonged inflammation and it frequently co-occurs with inflammatory arthritis [6]. IL-33 is one of the newly discovered members of inflammatory cytokines [6] and has been reported to trigger IgE-triggered release of IL-8 [7]. It is well established in literature that stress signals such as psoriasis prompts the release of $\mathrm{CRH}$ from the hypothalamus paraventricular nucleus (PVN). $\mathrm{CRH}$ in turn triggers $\mathrm{ACTH}$ release from anterior pituitary [8] which ultimately controls the glucocorticoid discharge from adrenal cortex. Several of the glucocorticoids, which include but are not limited to cortisol, at low doses trigger cutaneous immune and suppress the immune responses at high doses [9]. In the sympathetic nervous system, stress stimuli cause activation of the locus coeruleus (LC) of norepinephrine cells (NE) [10]. The neuropeptides which are products of the sympathetic response substance $P$ (SP), cutaneous nerve growth factor (NGF) and calcitonin gene-related peptide (CGRP), are reported to be pro- and anti-inflammatory depending on the type of the immune [11-13]. Moreover, it has been reported that $\mathrm{CRH}$ is tightly bound to a high affinity plasma binding protein (CRHBP) and CRHBP limits the distribution of $\mathrm{CRH}$ in the body [14].

In the current study, we examined the expression of $\mathrm{CRH}$ in psoriasis biopsy samples and correlated its expression with IL-8, IL-33 and CRHBP. The results revealed that expression of $\mathrm{CRH}$ is positively associated with expression of interleukin IL-8, IL-33 and negatively correlated with the expression of CRHBP.

\section{EXPERIMENTAL}

\section{Collection of samples}

Biopsies were taken from normal skin tissues of healthy volunteers as well as untreated psoriasis patients. The proper consent was given by the patients under protocols approved by the Institutional Review Board of Wuhan No.1 Hospital (no. 2016/IEC/S027). For immunohistochemistry analysis the samples were treated with formalin and paraffin embedded. The tissue samples were used for isolation of RNA, quantification protein expression levels of $\mathrm{CRH}, \mathrm{CRHBP}, \mathrm{IL}-8$ and IL33 as described previously $[15,16]$. The study was carried out after the ethical approval was provided by the ethical committee of Wuhan No.1 Hospital. The whole procedure was carried out according to international guidelines [17].
RNA isolation, synthesis of CDNA and expression analysis

Total RNA was isolated by RNeasy RNA isolation kit (Qiagen, China Shanghai Co Ltd) as per the manufacturer's protocol. Thereafter, cDNA was synthesized with the help of RevertAid cDNA synthesis kit (Fermentas) as per the guidelines provided by the manufacturer.

To carry out the quantification of the mRNA levels, the cDNA was first diluted at least 20 times and QRT-PCR was carried out thrice in ABI StepOne Real time (Applied biosystems), California, United States) using SYBR Green Master Mix (Fermentas, Massachusetts, United States). The relative quantification method $(\Delta \Delta$ CT) was employed to determine quantitative variation between the samples examined. $\beta$-Actin was used as positive control.

\section{Western blotting}

The skin tissues were lysed in lysis buffer and protein extracts were collected. Equal protein extracts from each group were run on SDS PAGE and then transferred to a polyvinylidene fluoride membrane. This was followed by blocking with $5 \%$ non-fat milk and incubation at $25^{\circ} \mathrm{C}$ for $1 \mathrm{~h}$.

Thereafter the membranes were administrated with a specific primary antibody at $4{ }^{\circ} \mathrm{C}$ overnight. This was followed by washing in washing buffer and incubation for $1 \mathrm{~h}$ with appropriate secondary antibody. The protein bands of interest were visualized with an ECL Advanced Western Blot Detection Kit.

\section{Immunohistochemistry}

For immunohistochemistry, monoclonal mouse anti-human antibodies to IL-33 and IL-8 were obtained from Invitrogen, Carlsbad, CA. The binding of the mouse antibody was demonstrated in skin biopsies with the help of biotinylated horse anti-mouse IgG and an avidin-biotin complex used with a peroxidase visualization reaction as per the guidelines provided by the manufacturer.

\section{Statistical analysis}

The data is presented as mean of three replicates \pm SD. GraphPad Prism 7 software was employed to carry out one way ANOVA followed by Tukey's post hoc test. The values were considered significant variously at ${ }^{*} p<0.01,{ }^{* *} p$ $<0.001$ and ${ }^{* * *} p<0.0001$. 


\section{RESULTS}

\section{Expression of $\mathrm{CRH}$ is highly induced in psoriasis}

It is well reported that expression of $\mathrm{CRH}$ is induced under stress conditions, therefore we evaluated the expression of $\mathrm{CRH}$ in healthy and psoriatic skin biopsy samples. The results revealed that expression of $\mathrm{CRH}$ was highly upregulated in all the psoriatic biopsy samples as compared to normal skin samples. The increase in the expression of $\mathrm{CRH}$ in psoriatic skin samples ranged from 2.7-3.5-fold (Figure 1).

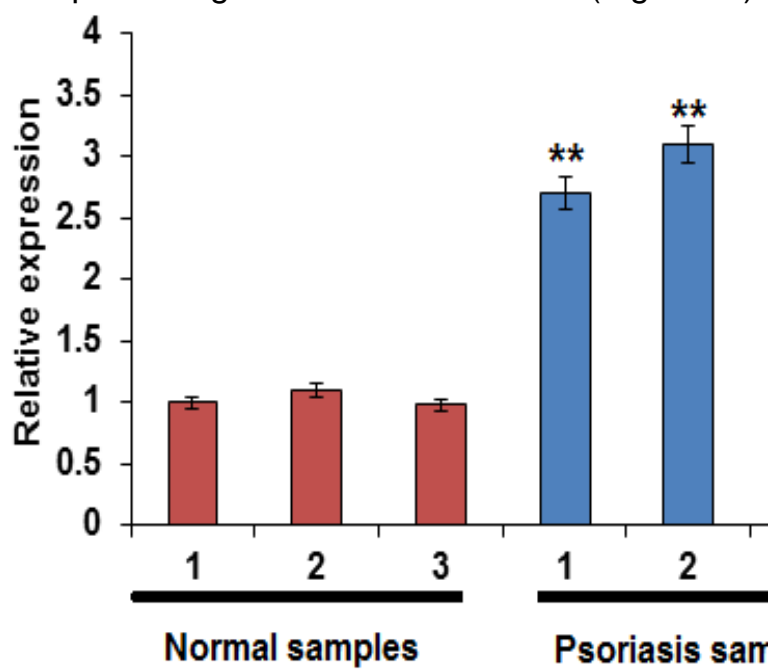

Figure 1: Expression of $\mathrm{CRH}$ in normal and psoriasis skin biopsy samples determined by semi-quantitative RT-PCR analysis. Experiments were carried out in three biological replicates and presented as mean \pm $\mathrm{SD}$. The values were considered significant at ${ }^{*} p<$ $0.01,{ }^{* *} p<0.001$ and ${ }^{* \star *} p<0.0001$

\section{Expression of CRH is associated with downregulation of CRHBP}

Studies have reported that $\mathrm{CRH}$ is tightly bound to CRHBP that limits its distribution [18]. Therefore, we evaluated the expression of CRHBP in psoriatic and normal skin biopsy samples by quantitative RT-PCR. The results of the present investigation revealed that the expression of CRHBP was significantly inhibited in the psoriatic samples in comparison to the normal skin samples.

The expression of CRHBP was 3.0 to 6.2 -fold lower in the psoriatic skin than in normal skin (Figure 2). To further, confirm this we evaluated the expression of CRHBP in one normal and one psoriatic skin biopsy samples by western blotting. The results of western blotting showed similar trend as that of RT-PCR analysis (Figure 3).

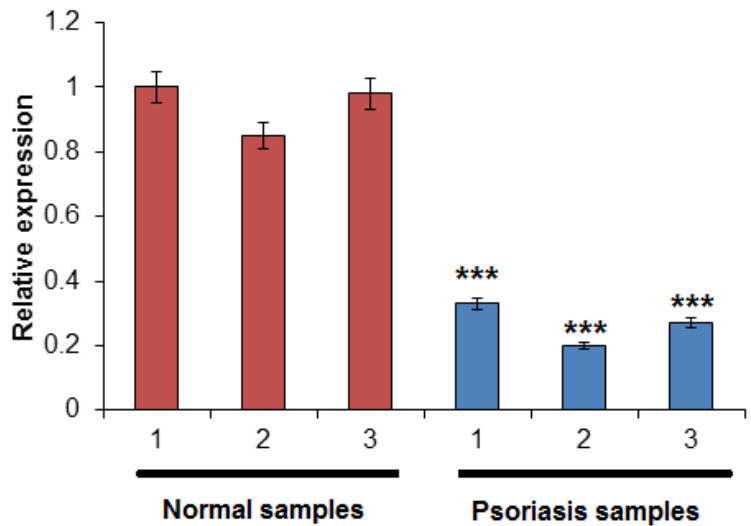

Figure 2: Expression of CRHBP in normal and psoriasis skin biopsy samples determined by semiquantitative RT-PCR analysis. Experiments were carried out in three biological replicates and presented as mean \pm SD. The values were considered significant at ${ }^{*} p<0.01,{ }^{* *} p<0.001$ and ${ }^{* * *} p<0.0001$
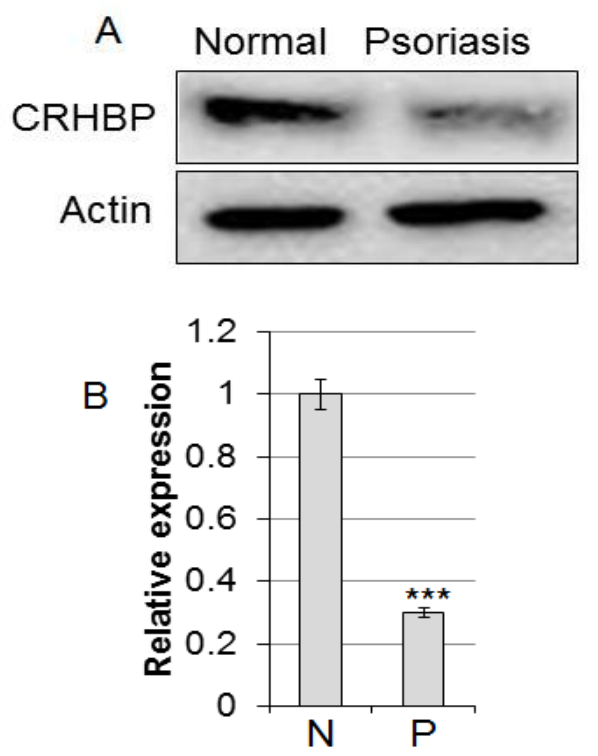

Figure 3: Protein expression of CRHBP in normal and psoriasis skin biopsy samples determined by western blot analysis. Experiments were carried out in three biological replicates and presented as mean \pm SD. The values were considered significant at ${ }^{*} p<0.01$, ${ }^{* *} p<0.001$ and ${ }^{* * *} p<0.0001$

\section{Expression of CRH is associated with upregulation of IL-8 and IL-33}

Psoriasis is one of the chronic inflammatory skin disorders and is always associated with prolonged inflammation [19]. In the current study, we examined the expression of inflammatory cytokines IL-8 and IL-33. The results showed that the expression of both IL-8 and IL-33 were significantly higher in psoriatic skin biopsy samples in comparison to the normal skin samples (Figure 4A-B).

The upregulated expression of IL-8 and IL-33was further confirmed by western blot in one psoriatic

Trop J Pharm Res, November 2017; 16(11): 2597 

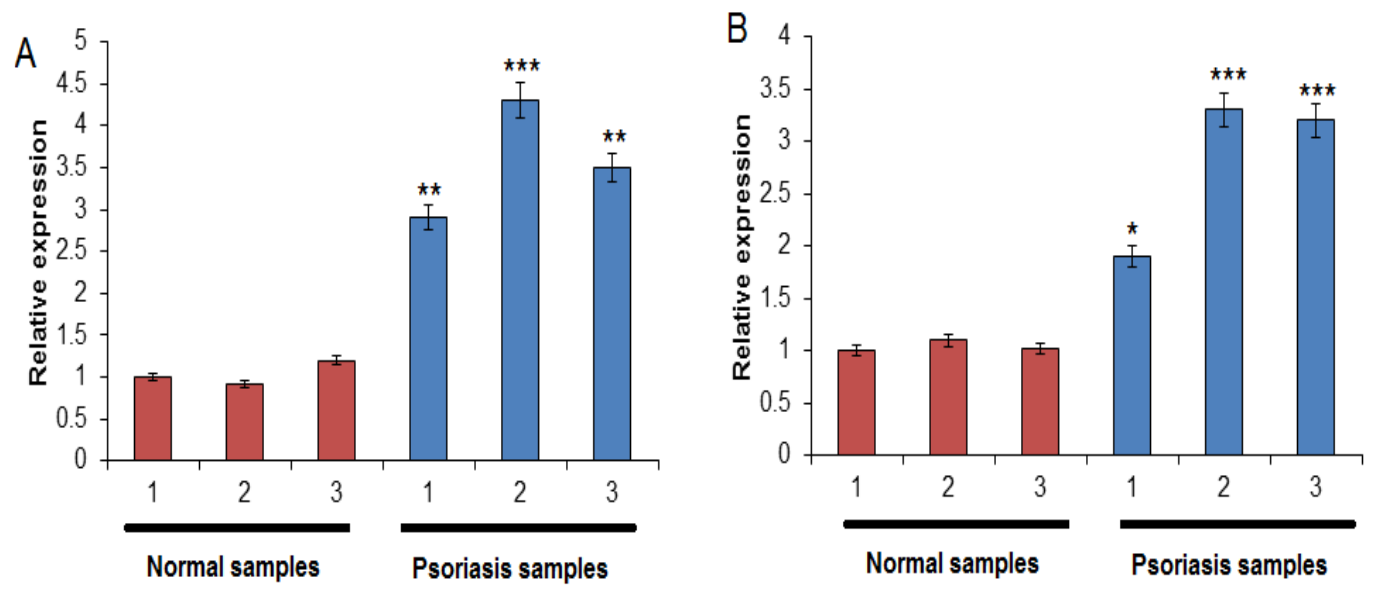

Figure 4: Expression of (A) IL-8 and (B) IL-33 in normal and psoriasis skin biopsy samples determined by semiquantitative RT-PCR analysis. Experiments were carried out in three biological replicates and presented as mean $\pm \mathrm{SD}$. The values were considered significant at ${ }^{*} p<0.01,{ }^{* *} p<0.001$ and ${ }^{* * *} p<0.0001$
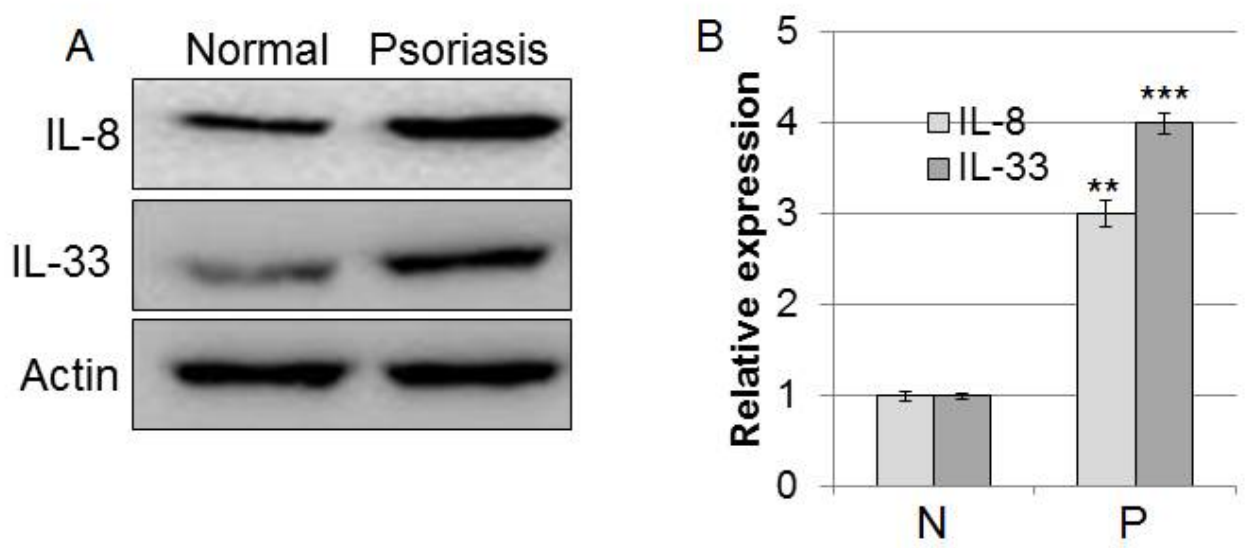

Figure 5: (A) Expression of IL-8 and IL-33 in normal and psoriasis skin biopsy samples determined by western blotting (B) quantification of IL-8 and IL-33 expression by densitometry. Experiments were carried out in three biological replicates and presented as mean \pm SD. The values were considered significant at ${ }^{*} p<0.01,{ }^{* *} p<$ 0.001 and ${ }^{* \star *} p<0.0001$
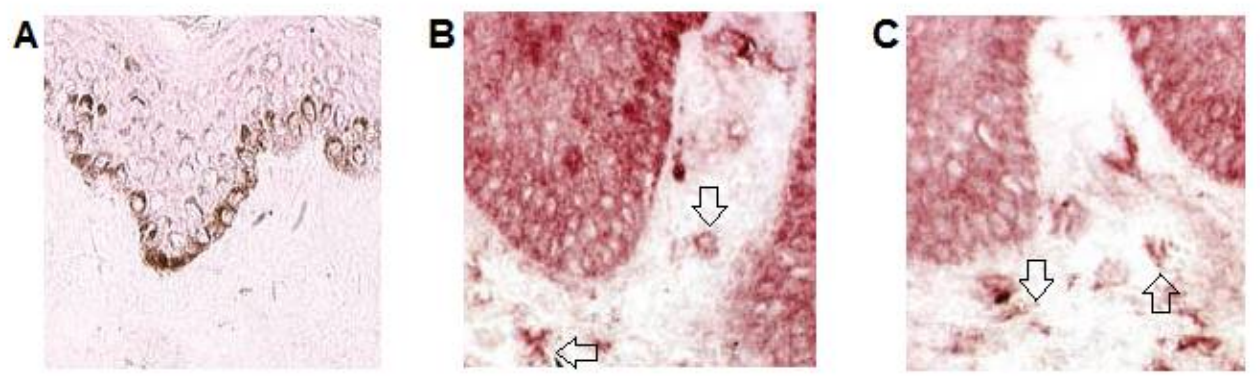

Figure 6: Immunohistochemistry showing distribution of IL-8 and IL-33 (A) normal skin biopsy sample showing no expression (B) psoriasis skin biopsy sample showing expression of IL-8 and (C) psoriasis skin biopsy sample showing expression of IL-33. Experiments were carried out in three biological replicates

and one normal skin samples and the protein expression of IL-8 and IL-33 correlated well with the results of RT-PCR data (Figure 5).

\section{Cell characteristics}

The results of immunohistochemistry revealed the presence of positively stained cells in psoriatic skin tissue indicating the expression of IL-8 and IL-33. However, no background staining was observed in case of the normal skin tissues (Figure 6A-C). 


\section{DISCUSSION}

Psoriasis affects $3 \%$ of the population around the world. In human, it is one of the prevalent $T$ cell arbitrated inflammatory diseases. A highly elevated expression of cytokines has been reported in psoriasis which include but are not limited to IL-1, IL-6 and IL-12 [20]. CRH is an important stress induced hormone that has been reported to trigger immune response indirectly [21].

In the current investigation, we examined the expression of $\mathrm{CRH}$ and correlated its expression with $\mathrm{CRHBP}, \mathrm{IL}-8$ and the newly included cytokine IL-33. CRH plays an important role in the hypothalamic pituitary adrenal (HPA) axis response to stress. At the same time, it also acts indirectly in an anti-inflammatory response. In the current study, it was observed that expression of $\mathrm{CRH}$ was highly induced in psoriatic skin biopsy samples (Figure 1). The results are in agreement with the studies carried out previously wherein the expression of $\mathrm{CRH}$ was reported to be highly induced of psoriatic samples.

There is concrete evidence that the distribution and function of $\mathrm{CRH}$ is limited by the presence of CRHBP. Therefore, we evaluated the expression of CRHBP in both normal and psoriatic skin biopsy samples (Figure 2). It was observed that the mRNA expression of CRHBP was significantly low in case of psoriatic samples. These results suggested that the higher expression of $\mathrm{CRH}$ in psoriatic skin biopsy samples may be due to the unavailability of CRHBP. The expression of CRHBP was further confirmed by examining its expression at the protein level by western blotting. The results of western blots confirmed the mRNA expression determined by quantitative RT-PCR. Furthermore, it is believed that cytokines could be possible therapeutic targets for the treatment of psoriasis [22].

In the present study, we determined the expression of IL-8 and the new cytokine IL-33 in both the psoriatic and normal skin samples. The results suggested that mRNA expression of both the cytokines was highly elevated. This expression of IL-8 and IL-33 was further confirmed by western blotting. The results are in confirmation with previous studies wherein expression of several cytokines such as IL-1, IL2, IL-6, IL-12 and IL-19 was found to be upregulated in psoriatic tissues [23]. While IL-8 was reported previously to be expressed in psoriasis [23], the present study represents one of the first studies that report the upregulation of IL-33 in psoriasis and IL-33 could therefore prove to be an important therapeutic target. Based on the results of immunostaining, we found that mononuclear leucocytes are possible sources of IL-33 in psoriatic lesions. Taken together, the results suggest that IL-33 antagonists may prove to be important treatment options for the treatment of diseases like psoriasis.

\section{CONCLUSION}

The findings of the present study reveal that the expression of $\mathrm{CRH}$ is up-regulated in psoriatic skin biopsy samples. This may be attributed to the down-regulation of CRHBP. Furthermore, the expression of $\mathrm{CRH}$ in psoriatic tissues is associated with concomitant accumulation of IL-8 and IL-33, indicating that $\mathrm{CRH}$ may trigger immune response in psoriasis by indirect stimulation of IL-8 and IL-33. Moreover, in view of the fact that $\mathrm{IL}-33$ is highly expressed in psoriasis, IL-33 antagonists may prove an appropriate treatment option for psoriasis.

\section{DECLARATIONS}

\section{Acknowledgement}

The authors acknowledge a grant from Scientific Research Project Foundation of Health and Family Planning Commission of Wuhan Municipality (NO.WX16B03).

\section{Conflict of Interest}

No conflict of interest associated with this work.

\section{Contribution of Authors}

The authors declare that this work was done by the authors named in this article and all liabilities pertaining to claims relating to the content of this article will be borne by them.

\section{Open Access}

This is an Open Access article that uses a funding model which does not charge readers or their institutions for access and distributed under the terms of the Creative Commons Attribution License (http://creativecommons.org/licenses/by/ 4.0) and the Budapest Open Access Initiative (http://www.budapestopenaccessinitiative.org/rea d), which permit unrestricted use, distribution, and reproduction in any medium, provided the original work is properly credited.

\section{REFERENCES}

1. Christophers E, Mrowietz U. Psoriasis. In: Freedberg IM, 
Eisen AZ, Wolff K, Austen KF, Goldsmith LA, Katz SI. Dermatology in General Medicine. New York: McGrawHill; 1999; $p 495$.

2. Nickoloff BJ. Creation of psoriatic plaques: the ultimate tumor suppressor pathway: a new model for an ancient T-cell-mediated skin disease: viewpoint. J Cutan Pathol 2001; 28:57-64.

3. Christophers E, Henseler T. Contrasting disease patterns in psoriasis and atopic dermatitis. Arch Dermatol Res 1987; 279(Suppl): S48-S51.

4. Oppmann B, Lesley R, Blom B, Timans JC, Xu Y, Hunte B, Vega F, Yu N, Wang J, Singh K, Zonin F. Novel p19 protein engages IL-12p40 to form a cytokine, IL-23, with biological activities similar as well as distinct from IL-12. Immunity 2000; 13: 715-725.

5. Parham C, Chirica M, Timans J, Vaisberg E, Travis M, Cheung J, Pflanz S, Zhang R, Singh KP, Vega F, To W. $A$ receptor for the heterodimeric cytokine $I L-23$ is composed of IL-12Rbeta1 and a novel cytokine receptor subunit, IL-23R. J Immunol 2002; 168: 5699-5708

6. Elkins KL, Cooper A, Colombini SM, Cowley SC, Kieffer $T L$. In vivo clearance of an intracellular bacterium, Francisella tularensis LVS, is dependent on the p40 subunit of interleukin-12 (IL-12) but not on IL-12 p70. Infect Immun 2002; 70: 1936-1948.

7. Wiekowski MT, Leach MW, Evans EW, Sullivan L, Chen SC, Vassileva G, Bazan JF, Gorman DM, Kastelein RA, Narula S, Lira SA. Ubiquitous transgenic expression of the IL-23 subunit p19 induces multiorgan inflammation, runting, infertility, and premature death. J Immunol 2001; 166: 7563-7570.

8. Chrousos GP. The Hypothalamic Pituitary Adrenal axis and the immune/inflammatory reaction. $N$ Eng $J$ Med 1995; 332: 1351-1362.

9. Johnson HM. Neuroendocrine peptide hormone regulation of immunity. Neuroimmunoendocrinology, end2nd, pp. 4983, Eds Karger, 1992.

10. Mastorakos G, llias I. The emerging role of peripheral corticotrophin releasing hormone (CRH). J Endocrinollnvest 2002, 26 364-371.

11. Chrousos GP, Gold PW. The concepts of stress and stress system disorders. Overview of physical and behavioural homeostasis. JAMA 1992; 267: 1244-1252

12. Cua DJ, Sherlock J, Chen YI, Murphy CA, Joyce B, Seymour B, Lucian L, To W, Kwan S, Churakova T, Zurawski S. Interleukin-23 rather than interleukin-12 is the critical cytokine for autoimmune inflammation of the brain. Nature 2003; 421: 744-7748
13. Austin LM, Ozawa M, Kikuchi T, Walters IB, Krueger JG. The majority of epidermal $T$ cells in psoriasis vulgaris lesions can produce type 1 cytokines, interferongamma, interleukin-2, and tumor necrosis factor-alpha, defining TC1 (cytotoxic T lymphocyte) and TH1 effector populations: a type 1 differentiation bias is also measured in circulating blood $T$ cells in psoriatic patients. J Invest Dermatol 1999; 113: 752-759.

14. Koo JY. Current consensus and update on psoriasis therapy: a perspective from the U.S. J Dermatol 1999; 26: 723-733.

15. Slominski A, Zbytek B, Semak I, Sweatman T, Wortsman J. CRH stimulates POMC activity and corticosterone production in dermal fibroblasts. J Neuroimmunol 2005; 162(1): 97-102.

16. Takematsu H, Tagami H. Quantification of chemotactic peptides (C5a anaphylatoxin and IL-8) in psoriatic lesional skin. Arch dermatol 1993;129(1): 74-80.

17. Troyer D. Biorepository standards and protocols for collecting, processing, and storing human tissues. Tissue Proteomics 2008: 193-220.

18. Krueger JG. The immunologic basis for the treatment of psoriasis with new biologic agents. J Am Acad Dermatol 2002; 46: 1-23.

19. Oestreicher JL, Walters IB, Kikuchi T, Gilleaudeau P, Surette J, Schwertschlag U, Dorner AJ, Krueger JG, Trepicchio WL. Molecular classification of psoriasis disease associated genes through pharmacogenomic expression profiling. Pharmacogenomics J 2001; 1: 272-287.

20. Bowcock AM, Shannon W, Du F, Duncan J, Cao K, Aftergut K, Catier J, Fernandez-Vina MA, Menter A. Insights into psoriasis and other inflammatory diseases from large-scale gene expression studies. Hum. Mol Genet 2001; 10: 1793-1805.

21. Zhou X, Krueger JG, Kao MC, Lee E, Du F, Menter A, Wong WH, Bowcock AM. Novel mechanisms of T-cell and dendritic cell activation revealed by profiling of psoriasis on the 63,100-element oligonucleotide array. Physiol Genomics 2003; 13: 69-78.

22. Yawalkar N, Karlen S, Hunger R, Brand CU, Braathen LR. Expression of interleukin-12 is increased in psoriatic skin. J Invest Dermatol 1998; 111:1053-1057.

23. Trepicchio WL, Ozawa M, Walters IB, Kikuchi $T$, Gilleaudeau P, Bliss JL, Schwertschlag U, Dorner AJ, Krueger JG. Dorner, J.G. Krueger. Interleukin-11 therapy selectively downregulates type I cytokine proinflammatory pathways in psoriasis lesions. J Clin Invest 1999; 104: 1527-1537. 